\title{
Herzinsuffizienz
}

\section{Pflanzlich basierte Nahrung wirkt protektiv}

\section{Eine überwiegend pflanzliche Ernäh- rung ist anderen Ernährungsmustern signifikant überlegen, was die Schutz- wirkung vor einer Herzschwäche betrifft, so das Ergebnis einer Kohor- tenstudie aus den USA.}

_ Die Autoren hatten prospektiv den Einfluss der Ernährung im Südosten der USA, dem sog. „,Schlaganfall-Gürtel“, in der Studie REGARDS (Reasons for Geographic and Racial Differences in Stroke), auf kardiovaskuläre Erkrankungen untersucht. Bei der vorliegenden Un- tersuchung, die auf Herzinsuffizienz fokussierte, wurden 15.569 gesunde Teilnehmer im Schnitt acht Jahre beobachtet. 300 von ihnen erhielten in dieser Zeit die Diagnose Herzinsuffizienz.

Erfasst wurde nun die Assoziation zu fünf Ernährungsmustern:

A Fertignahrungsmittel mit viel Fleisch, Nudeln, Fast-Food

B Pflanzliche Ernährung (reich an dunkelgrünem Blattgemüse, Obst, Bohnen, Vollkorn und Fisch)

C süße Diät (Brot, süßes Frühstück, Nachtische, Schokolade)
D Südstaaten-Diät (Eier, Frittiertes, Innereien, verarbeitetes Fleisch, Limonaden)

E Alkohol und Salat (Salate, Gemüse, Butter, Wein, Spirituosen).

Wer überwiegend auf Punkt b setzte, hat gewonnen: Die überwiegende Pflanzenkost reduzierte das Herzinsuffizienzrisiko gegenüber allen anderen Gruppen um $42 \%$. Da es sich um eine Beobachtungsstudie handelt, ist das Ursache-FolgePrinzip noch nicht bewiesen. - $\quad$ DE

- American Heart Association, Scientific Sessions 2017
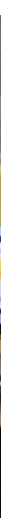

\section{Kaffee-Genuss senkt Risiken für Herzschwäche und Schlaganfall}

\section{Erneut gibt es gute Nachrichten für Kaffeetrinker: Hoher Kaffeekonsum stärkt das Herz und schützt das Hirn.}

Beim europäischen Herzkongress im August 2017 hatten spanische Autoren die Ergebnisse einer Studie berichtet, denen zufolge das relative Sterberisiko mit jeder täglich konsumierten Tasse Kaffee um 22\% abnahm. Beim US-Herzkongress im November 2017 kamen Forscher der Universität Colorado in Auro- ra nun zu dem Ergebnis, dass die relativen Risiken für Herzinsuffizienz und Schlaganfall mit jeder wöchentlich getrunkenen Tasse Kaffee um 7\% bzw. 8\% abnehmen.

Die US-Forscher hatten Daten der Framingham Heart Study, der Cardiovascular Heart Study und der Atherosclerosis Risk in Communities Studie zusammengebracht und sie in einem „Big-Data“-Ansatz von „lernenden Computern“ auswerten lassen („random fo- rest machine-learning methods"). Im Blick hatten sie Assoziationen zwischen Diätgewohnheiten und den Risiken für Herzinsuffizienz und Schlaganfall.

Dabei identifizierten sie die bekannten Risikofaktoren wie z. B. Alter, Cholesterin und Blutdruck sowie als einzigen Ernährungsfaktor mit deutlich protektiver Wirkung den regelmäßigen Kaffeekonsum.

- DE 